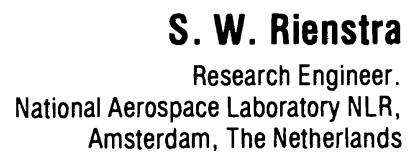

S. W. Rienstra Amsterdam, The Netherlands

\section{On the Acoustical Implications of Vortex Shedding from an Exhaust Pipe}

Asymptotic approximations for small Strouhal number are derived for the solution of the problem of the interaction between an acoustic wave and a subsonic jet flow issuing from a semi-infinite pipe. Density and sound speed differences between the jet flow and the (slowly moving) ambient medium, and a general edge condition are included. The approximations relate to the field inside the jet flow, to the far field, to the reflection coefficient, end-impedance and end correction for the reflected wave inside the pipe, and to the transmitted and radiated sound power. Within the range of parameters considered, the effect of the density and sound speed differences and ambient flow is found to be appreciable, although the character of the solution is not changed. However, the choice of the edge condition does have important implications; specifically, the phase of the reflected wave is most sensitive to only slight deviations from the Kutta condition.

\section{Introduction}

Only a few years ago, it was quite generally believed that a Kutta condition at a sharp trailing edge (effecting vortex shedding) in aero-acoustical problems may have some importance in that it changes radiation directivities with a few decibels or so, but never in such a dramatic way that orders of magnitude are involved ([9], p. 449; [23], p. 364). However, the experiments of Bechert, Michel and Pfizenmaier [2], together with Howe's [12] explanation, have shown this opinion not to be true. In the configuration of a subsonic jet issuing from a pipe perturbed from inside by long sound waves, they found that only a small fraction of the net sound power, transmitted through the pipe, was recaptured in the far field. The rest was transformed into hydrodynamic energy of vortices shed from the pipe edge. These vortices, making only little noise, arise from viscous and nonlinear action, which is, in an inviscid linear problem, modeled by an appropriate edge condition, like the Kutta condition. Reductions up to $25 \mathrm{~dB}$ were obtained, so in this case the Kutta condition really provides an important sink of sound. (The opinion that in general the Kutta condition and vortex shedding gives a reduction is not correct; at least in the case of an airfoil trailing edge there is, under some conditions, more sound radiated by the vortices interacting with the edge than was used for their production, and the net result is an amplification [21].) Various technological applications exploiting this mechanism for noise reduction are in use or under development [1], e.g., tube ends consisting of many parallel small nozzles, acoustic liners of Helmholtz resonators with through-flow, and exhaust mufflers for piston engines, so a deeper study of various aspects of this process seems well worth doing.

Contributed by the Noise Control and Acoustic National Group of THE American Society of Mechanical EngineERs and presented at the Winter Annual Meeting, November 16-21, 1980, Chicago, Illinois. Manuscript received at ASME Headquarters July 1980. Paper No. 80-WA/NC-16.
We can model in the foregoing problem the medium as inviscid, the pipe as semi-infinite, the jet flow with a uniform "top hat" velocity profile, and the shear layer by a vortex sheet. For this relatively simple model problem an acoustically exact solution is known, found by Munt [17]. This solution describes far field directivity [17], reflection coefficient at the pipe exit [18], and indeed, the above sound absorption [12] is in very good agreement with the experiments of $[2,19,24]$. Munt's solution is, however, constrained by the Kutta condition, and a causality condition that the source be switched to a long but finite time ago. This causality condition - a heritage from doubly infinite vortex sheet problems - involves complicated mathematics, and is not always satisfied for any edge condition. It therefore seemed to have hindered Munt's solution and the physics behind from being well interpreted and understood. Things are, however, much more simple. In fact, this condition of causality is not relevant and not necessary, as we explained in previous work $[20,22]$. The Kutta condition is, generally speaking, just sufficient to insure uniqueness of the solution. (To avoid confusion: the causality of the real physical system is of course beyond doubt; what we mean here is a basically mathematical condition adopted in the simplified model.) Briefly summed up, the arguments for this claim are as follows.

In the case of the related two-dimensional problem without solid body (a doubly infinite plane vortex sheet separating uniform subsonic flow from stagnant flow, subject to acoustic irradiation from a harmonic line source), the otherwise nonunique solution (since the amplitude of the Helmholtz instability is undetermined) can be rendered unique by assuming the source to be switched on ahead of time [13]. However, as compared to reality, it is very unlikely that the early start of the source should be decisive for the present state of the process. So many other things are not 
included in the model (a solid body, viscosity, nonlinearities, etc.), and could very well be responsible for the nonuniqueness of the solution. Indeed, if we extend the model to include a semi-infinite plate with a trailing edge, the edge condition is just sufficient for uniqueness [20]. Only in the presence of a trailing edge is the acoustic field at any instant coupled to the vortex sheet instability through the viscous forces working at the edge ( $\rightarrow$ edge condition); to remove the acoustically induced singularity, vorticity is shed from the edge into the plane of the vortex sheet, triggering the instability (see also [7], pp. 7, 8). The conclusion is that (in the inviscid model) any edge condition can be satisfied by simply adding a multiple of the Helmholtz unstable eigenmode.

All this was not realized at the time when the plate/vortex sheet problem [8, 16] and the pipe/vortex sheet problem [17] was solved, and much unnecessary emphasis was placed by these authors on the causality condition.

Several aspects of the issue discussed could be worked out in more detail, but these are of a more technical nature and outside the scope of the present paper. For further information, the interested reader is referred to the literature mentioned.

To exploit Munt's solution further-which is, although exact, of a complicated form, and has to be evaluated numerically-asymptotic approximations for small Strouhal number were carried out by Cargill [5] and Rienstra [20, 22]. The latter studied the acoustic field inside the jet flow for a cold jet without coflow, subject to two edge conditions, viz., the Kutta condition and the condition of no vortex shedding. The former investigated the ambient far field and the (modulus of the) reflection coefficient inside the pipe for a cold or hot jet with coflow and the Kutta condition applied; unfortunately, however, due to space limitations, his paper contained no indication as to which method was employed and which additional restrictions on the parameters are necessary. Cargill found that his simple far field formula, in the case of a cold jet, compares well with both Munt's calculations and the experimental results of Pinker and Bryce [19], and also that the ratio of transmitted and radiated sound power agrees with Bechert, et al.'s [2] experiments, displaying again Howe's acoustic energy conversion mechanism.
The purpose of the present paper is to extend these previous contributions to include sound speed, density, and ambient flow effects on the field inside the jet flow, and to investigate the effect of deviations from the Kutta condition on the whole field.

Although we may expect the Kutta condition to be valid in many cases, there is little doubt that sometimes it is not satisfied [2, 4, 11]. Among other factors, an increasing amplitude or frequency will produce an edge condition gradually deviating from the Kutta condition, in a way that less vorticity (and probably with another phase) is shed than with the full Kutta condition. It is not clear a priori how this will affect the acoustics and whether it explains some discrepancies, like those between Munt's calculations for a hot jet [17] and the experiments by Pinker and Bryce [19].

The results are presented with the most general edge condition compatible with the acoustics. Any particular edge condition can be selected afterwards; for instance, all the previous results mentioned herein before are automatically included. The formulas obtained are valid asymptotically for small Strouhal number and uniformly for a subsonic jet Mach number not close to one. The other parameters are somewhat restricted but not severely. The paper concludes with a discussion concerning the relation between the present analysis and experiments.

\section{Formulation of the Problem}

Consider a semi-infinite circular pipe with diameter $D$ from which a subsonic jet issues into a medium, which may be stagnant or may flow in the same direction as, but slower than, the jet (Fig. 1). The jet flow is perturbed by plane harmonic sound waves of frequency $f^{*}$ coming from inside the pipe. Viscosity, thermal conductivity, and all nonlinearities will be ignored. The stationary velocity of the jet, $U_{j}$, and of the fluid outside, $U_{o}$, are uniform (i.e., constant in space). Mean pressure $p_{0}$ is the same everywhere, and mean density and sound speed inside and outside the jet are denoted $\rho_{j}, c_{j}, \rho_{o}$, and $c_{o}$, respectively. The Mach number of the jet is $\mathbf{M}_{j}=u_{j} / c_{j}$, and of the fluid outside $\mathbf{M}_{o}=$ $U_{o} / c_{o}$, with $0 \leq \mathbf{M}_{o}<\mathbf{M}_{j}<1$. The cylinder is specified by $r^{*}=1 / 2 D, z^{*} \leq 0$ in cylindrical polar coordinates $\left(r^{*}, \theta, z^{*}\right)$,

\section{Nomenclature}

$$
\begin{aligned}
a & =\text { amplitude } \\
A_{0} & =\text { equation }(10) \\
c_{j}, c_{o} & =\text { soundspeed in/outside } \\
C & \left.=c_{j} / c_{o}\right) \\
C_{J} & =0.2554 . . \\
d & =\rho_{o} / \rho_{j} \\
D & =\text { pipe diameter (dim.) } \\
D_{v} & =\text { equation }(18) \\
E_{v} & =\text { equation }(19) \\
f^{*} & =\text { frequency (dim.) } \\
F_{+} & =\text {equation }(9) \\
h & =\text { vortex sheet displacement; } \\
& \text { equation }(1) \\
\mathrm{H}_{m}^{(2)} & =\text { Hankel function } \\
i & =\text { imaginary unit } \\
\mathrm{Im} & =\text { imaginary part } \\
j_{m, n} & =n^{\text {th }} \text { zero of } J_{n}(x) / x^{m} \\
J_{m} & =\text { Bessel function } \\
k & =\omega \mathrm{M}_{j} ; \text { Helmholtz number } \\
l & =\text { end correction } \\
L & =-1 / 2 \ln \left(1 / 2 \omega \beta_{c} e^{\gamma}\right) \\
\mathrm{M}_{j}, \mathrm{M}_{o} & =\text { Mach number in/outside } \\
N_{+} & =\text {equation }(11)
\end{aligned}
$$

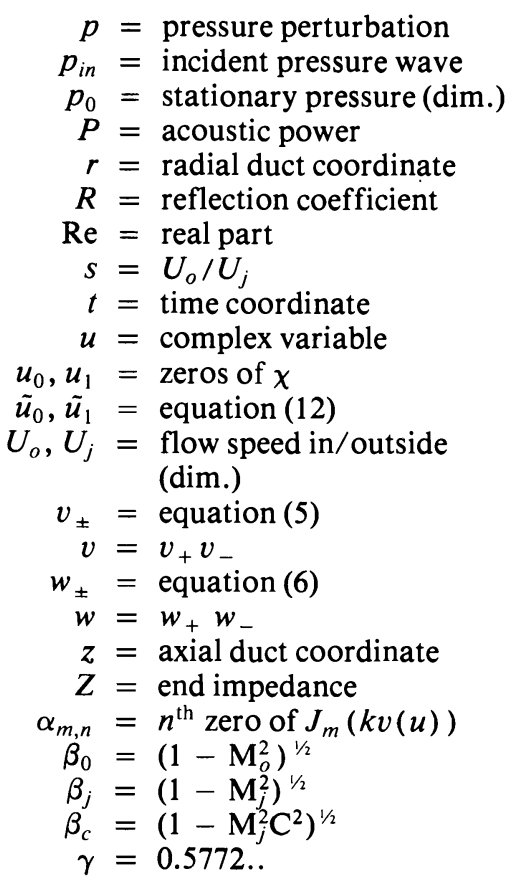

$$
\begin{aligned}
\Gamma & =\text { edge condition parameter } \\
\zeta & =\text { equation }(17) \\
\Theta & =\text { equation }(20) \\
\lambda & =\left(r^{2}+z^{2} \beta_{0}^{-2}\right)^{1 / 2} / \beta_{0} \\
\mu & =\chi(u) /\left(u-u_{0}\right)\left(u-u_{1}\right) \\
\mu_{ \pm} & =\text {equation }(8) \\
v & =0,1 ; \text { index } \\
\xi & =\text { arctan }\left(\beta_{0} r / z\right) \\
\xi_{0} & =\text { instability cone angle } \\
\pi & =3.1416 . . \\
\rho & =\text { density perturbation } \\
\rho_{j}, \rho_{0} & =\text { stationary density } \\
& \text { in/outside }(\text { dim. }) \\
\sigma_{ \pm} & =\text {min }\left[\left(1 \pm \mathrm{M}_{j}\right)^{-1},\right. \\
\Sigma_{v} & \left.=C\left(1 \pm \mathrm{M}_{o}\right)^{-1}\right] \\
\phi & =\text { equation }(21) \\
\chi & =\text { equation }(7) \\
\psi & =\text { potential; equation }(2) \\
\omega & =\pi f^{*} D / U_{j} ; \text { Strouhal } \\
& \text { number }
\end{aligned}
$$

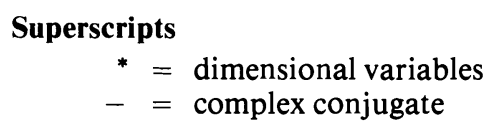

* = dimensional variables

- = complex conjugate 
and the undisturbed vortex sheet, between the jet and the ambient flow, is given by $r^{*}=1 / 2 D, z^{*}>0$. The pressure and density perturbations are denoted by $p^{*}$ and $\rho^{*}$, respectively, and for the velocity perturbations, we introduce a potential $\phi^{*}$. The time variable is $t^{*}$. The mean quantities inside and outside are related by

$$
U_{o}=s U_{j}, \rho_{o}=d \rho_{j}, c_{o}=c_{j} / C, \mathrm{M}_{o}=s C \mathrm{M}_{j} .
$$

We make dimensionless variables as follows.

$r=r^{*} / 1 / 2 D, \quad z=z^{*} / 1 / 2 D, \quad t=t^{*} U_{j} / 1 / 2 D, \quad \phi=\phi^{*} / 1 / 2 D U_{j}$, $p=p^{*} / \rho_{j} U_{j}^{2}, \rho=\rho^{*} / \rho_{j}, \omega=\pi f^{*} D / U_{j}, k=\pi f^{*} D / c j=\omega \mathrm{M}_{j}$

(Note the factor $\pi$ in Strouhal number $\omega$ and Helmholtz number $k_{\text {.) }}$

Finally, we will assume $\omega$ to be small. How small will be sufficient is not known a priori; there is evidence that of several physical quantities the formulas calculated for small $\omega$ cover well the experimental data even for $\omega$ of order 1 , while on the other hand for some other quantities, there is a lack of agreemeent which might be attributed to a not sufficiently small $\omega$.

As usual, we write the dependent variables (like $\phi$ and $p$ ) in complex form, and it is tacitly understood that real parts should be taken. Then the primary pressure wave $p_{\text {in }}$, coming from inside the pipe, is given by

$$
\begin{array}{rlrl}
p_{\mathrm{ln}}(r, \theta, z, t) & =a p_{\mathrm{in}}(z) \exp (i \omega t) & \\
& =a \exp \left(-i \frac{k}{1+\mathrm{M}_{j}} z+i \omega t\right) & & (r<1), \\
& =0 & & (r>1)
\end{array}
$$

The dimensionless position of the vortex sheet is given by

$$
r=1+a h(z) \exp (i \omega t) \quad(z>0)
$$

The dimensionless amplitude $a$ is taken to be small enough for linearization. Furthermore, we assume the field induced by $p_{\text {ln }}$ to behave the same as $p_{\text {in }}$ with respect to time $t$ and angle $\theta$. So here it is independent of $\theta$ and having the factor $\exp (i \omega t)$; this factor, as well as the amplitude $a$, will be suppressed throughout. For convenience we write

$$
\phi(r, z)=i \frac{1+\mathrm{M}_{j}}{\omega} p_{\text {in }}(z)+\psi(r, z)
$$

so that we have for pressure (an index $z$ or $r$ denotes differentiation with respect to that variable)

$$
\begin{aligned}
p & =p_{\text {in }}-i \omega \psi-\psi_{z} & & (r<1), \\
& =\quad-d\left[i \omega \psi+s \psi_{z}\right] & & (r>1)
\end{aligned}
$$

The equations for $\psi$ are forms of the convected Helmholtz equation.

$$
\psi_{z z}+\frac{1}{r}\left(r \psi_{r}\right)_{r}+k^{2} \psi-2 i k \mathrm{M}_{j} \psi_{z}-\mathrm{M}_{j}^{2} \psi_{z z}=0 \quad(r<1),
$$

$\psi_{z z}+\frac{1}{r}\left(r \psi_{r}\right)_{r}+k^{2} C^{2} \psi-2 i k C \mathrm{M}_{o} \psi_{z}-\mathrm{M}_{o}^{2} \psi_{z z}=0 \quad(r>1)$

Finally, the boundary conditions are as usual (where $1+, 1-$ mean upper, lower limit),

$$
\begin{array}{ll}
\psi_{r}(1, z)=0 & (z<0), \\
p(1+, z)=p(1-, z) & (z>0), \\
\psi_{r}(1-, z)=i \omega h(z)+h_{z}(z) & (z>0), \\
\psi_{r}(1+, z)=i \omega h(z)+\operatorname{sh}_{z}(z) & (z>0), \\
\Psi(0, z)<\infty &
\end{array}
$$

$\psi(r, z)$ radiating outwards at infinity (in particular $r \rightarrow \infty$ ).

The edge condition is most conveniently made explicit by a characterization of the deflection of the vortex sheet near the edge. In general, we have

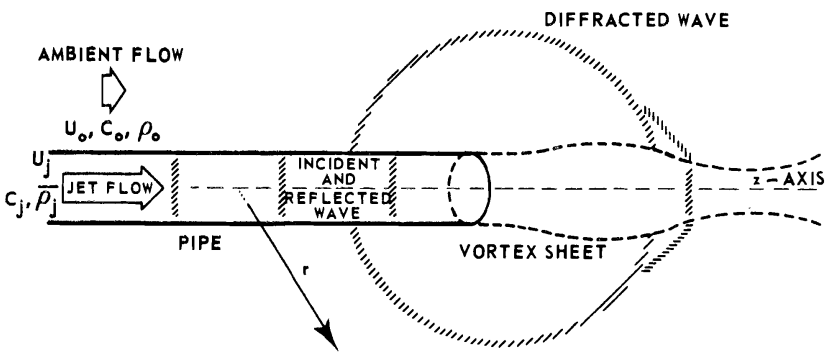

Fig. 1 Sketch of the problem

$$
h(z) \simeq(1-\Gamma)\left(H_{1} z^{1 / 2}+H_{2} z^{3 / 2}\right)+H_{3} z^{3 / 2}
$$

were $H_{1}, H_{2}$ and $H_{3}$ are constants, and $\Gamma$ is a complex valued parameter, controlling (indirectly) the amount of shed vorticity, and to be chosen in a way to meet the edge condition required in a given situation. $\Gamma$ has been defined such that $\Gamma=1$ corresponds to the Kutta condition (the flow leaves the edge tangentially), and $\Gamma=0$ corresponds to no vortex shedding. Anticipating the appearance of an instability by shed vorticity, it is sufficient for uniqueness to require additionally:

if $h(z) \simeq H_{1} z^{1 / 2}(z ! 0)$, then also $h(z) \rightarrow 0(z \rightarrow \infty)$.

\section{Exact Formal Solutions}

The solutions we are looking for are, in essence, a linear combination of Savkar's [23] (stable, no vortex shedding) and Munt's [17] (with Kutta condition) solutions. The difference is in fact one of Crighton's [6] eigensolutions, slightly generalized. To consider the issue of causality as was done by Munt is not necessary (neither relevant) as we argued before, so we will merely present the final formal solution here.

Consider in the complex $u$-plane the principal branch square roots

$$
\begin{gathered}
v_{+}(u)=\left(1-\left(1+\mathrm{M}_{j}\right) u\right)^{1 / 2}, \\
v_{-}(u)=\left(1+\left(1-\mathrm{M}_{j}\right) u\right)^{1 / 2}, \\
w_{+}(u)=\left(C-\left(1+\mathrm{M}_{o}\right) u\right)^{1 / 2}, \\
w_{-}(u)=\left(C+\left(1-\mathrm{M}_{o}\right) u\right)^{1 / 2},
\end{gathered}
$$

with

$$
v_{ \pm}(0)=w_{ \pm}(0) \mathrm{C}^{-1 / 2}=1
$$

and branch cuts running along the real axis to infinity not via the origin. Furthermore these square roots define $v=v_{+} v_{-}$ and $w=w_{+} w_{-}$. The functions $\chi(u)$ and $\mu(u)$, most important in the analysis, are defined as

$$
\begin{aligned}
\chi(u)=w(u)\left(1-\mathrm{M}_{j} u\right)^{2} \frac{J_{o}(k v(u))}{J_{1}(k v(u))} \\
-d v(u)\left(1-s \mathrm{M}_{j} u\right)^{2} \frac{H_{0}^{(2)}(k w(u))}{H_{1}^{(2)}(k w(u))} \\
=\left(u-u_{0}\right)\left(u-u_{1}\right) \mu(u)
\end{aligned}
$$

where $J_{n}$ is the $n^{\text {th }}$ order Bessel function of the first kind, and $H_{n}^{(2)}$ the $n^{\text {th }}$ order Hankel function of the second kind [25]. Two of $\chi$ 's zeros $\left(u_{0}\right.$ and $\left.u_{1}\right)$ are taken apart for convenience. They correspond to the Helmholtz instability of the vortex sheet $\left(u_{0}\right.$, first quadrant) and a related decreasing mode $\left(u_{1}\right.$, fourth quadrant), and they can be identified via their behavior for $\omega \rightarrow 0, \mathrm{M}_{j} C \rightarrow 0, d=O(1)$; then

$$
u_{0} \approx \bar{u}_{1} \simeq \mathrm{M}_{j}^{-1}\left(1+i \sqrt{1 / 2 d}(1-s) \omega \ln ^{1 / 2} \omega^{-1}\right)
$$

where the bar means complex conjugate. We introduce the functions (split functions) $\mu_{+}$and $\mu_{-}$such that $\mu=\mu_{+} / \mu_{-}$, and obeying certain regularity properties (see Munt). These functions are unique, modulo an entire function without zeros, and can be expressed as follows. Define, in the complex 
$u$-plane, a contour close to the real axis, just below the branch cuts at the negative side and just above at the positive side, and crosing the real axis somewhere between $\sigma_{+}$and $-\sigma_{-}$, where

$$
\sigma_{ \pm}=\min \left[\left(1 \pm \mathrm{M}_{j}\right)^{-1}, C\left(1 \pm \mathrm{M}_{o}\right)^{-1}\right]
$$

For a point $u$ not on this contour, $\mu_{+}(u)$ is given by

$\ln \mu_{+}(u)=\frac{1}{2 \pi i} \lim _{A \rightarrow \infty} \int_{-A-i .0}^{A+i .0} \frac{\ln \mu\left(u^{\prime}\right)}{u^{\prime}-u} d u^{\prime}+\epsilon(u) \ln \mu(u)$

where the integration runs along the above contour, and $\epsilon(u)$ $=0(\epsilon(u)=1)$ for $u$ above (below) the contour.

The Fourier transform $F_{+}(u)$ of $h(z)$, i.e.,

$$
F_{+}(u)=\int_{0}^{\infty} h(z) \exp (i k u z) d z
$$

is found to be

$$
F_{+}(u)=A_{0} N_{+}(u)\left\{\Gamma\left(u-u_{0}\right)^{-1}-\left(u-\frac{1}{1+\mathrm{M}_{j}}\right)^{-1}\right\}
$$

where

$$
\begin{array}{r}
A_{0}=\frac{i}{\omega^{2}} v_{-}\left(\frac{1}{1+\mathrm{M}_{j}}\right) w_{-}\left(\frac{1}{1+\mathrm{M}_{j}}\right) \mu_{-} \\
\left(\frac{1}{1+\mathrm{M}_{j}}\right)\left(u_{0}-\frac{1}{1+\mathrm{M}_{j}}\right)^{-1} \\
N_{+}(u)=\frac{v_{+}(u) w_{+}(u)}{\left(u-u_{1}\right) \mu_{+}(u)}
\end{array}
$$

The formal acoustically exact solution with arbitrary edge condition is then formulated as follows.

$$
\begin{aligned}
& r<1: \psi(r, \mathrm{z})= \\
& \frac{\omega}{2 \pi i} \oint_{-\infty-i .0}^{\infty+i .0} \frac{\left(1-u \mathrm{M}_{j}\right) J_{0}(k v(u) r) F_{+}(u)}{v(u) J_{1}(k v(u))} \exp (-i k u z) d u, \\
& r>1: \psi(r, z)= \\
& \frac{\omega}{2 \pi i} \oint_{-\infty-i .0}^{\infty+i .0} \frac{\left(1-u s \mathrm{M}_{j}\right) H_{0}^{(2)}(k w(u) r) F_{+}(u)}{w(u) H^{(2)}(k w(u))} \exp (-i k u z) d u
\end{aligned}
$$

The contour of integration is the same as defined for $\mu_{+}$, but with an indentation into the first quadrant surrounding the pole $u=u_{0}$ (see Fig. 2).

For $\Gamma=0$, no vorticity is shed by the incident sound wave, no instability is triggered, and indeed $F_{+}$has no pole in $u=$ $u_{0}$, so in this case the indentation around $u_{0}$ can be ignored.

The field inside the pipe $(r<1$ and $z<0)$ can be written more explicitly by closing the contour via a large semicircle in the upper half plane, and noting hat $F_{+}$is analytic above the contour of Fig. 2, and that $J_{0}(k v r)$ and $v J_{1}(k v)$ depend on $u$ through $v^{2}(u)$. Hence the field is given by a sum of residues in the zeros of $v(u) J_{1}(k v(u))$ : the usual modal expansion. This expansion will be given later in approximated form.

An asymptotic expression of the field for $k\left(r^{2}+z^{2}\right)^{1 / 2} \rightarrow \infty$ (the far field) can be obtained with the method of stationary phase. Following Munt we find then, after defining

$$
\begin{aligned}
& \beta_{0}=\left(1-\mathbf{M}_{0}^{2}\right)^{1 / 2}, \\
& r=\beta_{0} \lambda \sin \xi, \\
& z=\beta_{0}^{2} \lambda \cos \xi,
\end{aligned}
$$

for $k \lambda \rightarrow \infty$ and $\xi$ not near 0 or $\pi$,

$$
p(r, \mathrm{z}) \simeq
$$

$$
\begin{array}{r}
\frac{-i \omega d\left(1-\mathrm{M}_{0} \cos \xi\right)^{2} F_{+}\left(C \beta_{0}^{-2}\left(\cos \xi-\mathrm{M}_{0}\right)\right)}{\pi \beta_{0}^{5} C \mathrm{M}_{j} \lambda \sin \xi H_{1}^{(2)}\left(k C \beta_{0}^{-1} \sin \xi\right)} \\
\exp \left[-i k C \lambda\left(1-\mathrm{M}_{0} \cos \xi\right)\right]
\end{array}
$$

Inside the cone $0<\xi \leq \xi_{0}=$

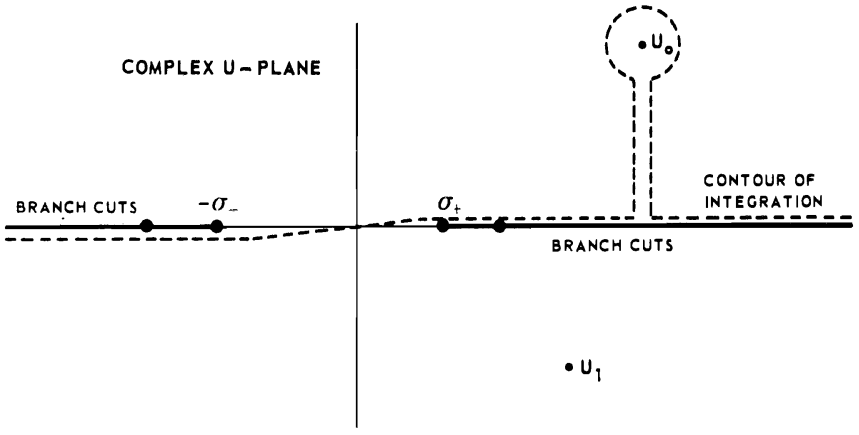

Fig. 2 Contour of integration

$\arccos \left[1 / 2 \sqrt{2}\left[1+\left|a_{0}\right|^{2}-\left\{\left(\left|a_{0}\right|^{2}-1\right)^{2}+4\left(\operatorname{Im} a_{0}\right)^{2}\right\}^{1 / 2}\right]^{1 / 2}\right]$ where $a_{0}=\beta_{0}^{2} C^{-1} u_{0}+\mathrm{M}_{0}$, the instability field, due to the contribution of the pole in $u=u_{0}$, should be included (if $\Gamma \neq$ $0)$. So there the present expression is formally not valid. There is, however, evidence that in the real world this instability is not so important, since it breaks up into acoustically less important eddies, and that the present expression is a reasonable representation of the far field also for $0<\xi \leq \xi_{0}$. (This has nothing to do, of course, with the acoustical importance of the interaction between the instability and the pipe edge.) Furthermore, in the limit $\omega \rightarrow 0$ we will consider, the cone becomes small enough to ignore. So further on, the instability wave will be neglected in the far field.

\section{Approximations for Small Strouhal Number}

In this section, we will present asymptotic approximations for $\omega \rightarrow 0$ to several physical quantities, including an estimate of the error. The method employed consists of a deformation of the contour of integration in such a way that on the new contour an approximation of the integrand, with a uniform relative error, is available, sufficiently simple to integrate explicitly. Details of the method can be found elsewhere [20, 22].

Beside $\omega$ we have several other parameters with, a priori, unknown magnitude with respect to $\omega$. Too much variation of these parameters may introduce the need to consider many special cases to be dealt with separately, and may even invalidate any approximation of the present kind. The number of possibilities is too large to explore all extensively, and we will therefore restrict the respective domains a bit as follows (in many "sound in air"' cases, these restrictions are met).

$$
\begin{gathered}
0<M_{j}<1, \text { provided } \beta_{j}=\left(1-\mathrm{M}_{j}^{2}\right)^{1 / 2}=O(1), \\
0 \leq s \leq O\left(\omega \ln ^{1 / 2} \omega^{-1}\right), \\
C=O(1), \text { provided } \mathrm{M}_{j} C<1, \text { and } \beta_{c}=\left(1-\mathrm{M}_{j}^{2} C^{2}\right)^{1 / 2}=O(1), \\
0<d \leq O(1), \\
|\Gamma| \leq 1
\end{gathered}
$$

The "order" symbol related to the limit $\omega \rightarrow 0$.

Now we can construct approximations to $\mu_{+}, \mu_{-}, u_{0}$ and $u_{1}$ with a relative accuracy of $O\left(\omega^{2} \ln \omega^{-1}\right)$, to find:

$$
\begin{aligned}
& u_{0} \simeq \tilde{u}_{0}=\mathrm{M}_{j}^{-1}(1+i \omega \sqrt{d L}), \\
& u_{1} \simeq \tilde{u}_{1}=\mathrm{M}_{j}^{-1}(1-i \omega \sqrt{d(L-1 / 2 \pi i))}
\end{aligned}
$$

with $L=-1 / 2 \ln \left(1 / 2 \omega \beta_{c} e^{\gamma}\right), \gamma=0.5772 \ldots$ (Euler's constant), and the principal branch of the square root; on a contour, deformed sufficiently away from $u= \pm C$ and $u=M_{j}^{-1}$,

$$
\begin{aligned}
& \mu(u) \simeq \frac{\left(1-u \mathrm{M}_{j}\right) w(u) J_{0}(k v(u))}{\left(u-\tilde{u}_{0}\right)\left(u-\tilde{u}_{1}\right) J_{1}(k v(u))}, \\
& \mu_{+}(u) \simeq \frac{\left(u-\mathbf{M}_{j}^{-1}\right)^{2} w_{+}(u)}{\left(u-\tilde{u}_{0}\right)\left(u-\tilde{u}_{1}\right) v_{+}(u)} \prod_{n=1}^{\infty} \frac{u-\bar{\alpha}_{0, n}}{u-\bar{\alpha}_{1, n}} \cdot \frac{j_{1, n}}{j_{0, n}}
\end{aligned}
$$


where

$$
\alpha_{m, n}=\frac{i}{\beta_{j} k}\left\{j_{m, n}^{2}-\left(\frac{k}{\beta_{j}}\right)^{2}\right\}^{1 / 2}-\frac{\mathbf{M}_{j}}{\beta_{j}^{2}}
$$

and $j_{m, n}$ is the $n^{\text {th }}$ zero of $J_{m}(x) / x^{m}$. Furthermore, the present method with contour deformation involves an error exponentially depending on $z$. A little more detailed analysis reveals that upstream this error can be ignored, but downstream an additional restriction is required, giving

$$
-\infty<z \leq O\left(\omega^{-1}\right)
$$

effectively meaning that in the jet the instability field is not very well included.

Finally, these approximations yield an approximation to the integrand of $\psi$ in $r<1$ from which the physical quantities can be calculated by a straightforward summation of residues. Also, these approximations are applicable to the far field formulas. And so, after some algebra, ignoring all the terms smaller than the error, we obtain for $r<1$ (in the jet flow):

$$
\begin{aligned}
& p(r, z \geq 0)=D_{0}\left(r, z / \beta_{j}\right)+O\left(\omega^{2} \ln \omega^{=1}\right) \\
& p(r, z \leq 0)=\exp \left(-\frac{i k}{1+\mathrm{M}_{j}} z\right) \\
& -\frac{1+\mathrm{M}_{j}-2 \mathrm{M}_{j} \Gamma}{1-\mathrm{M}_{j}} \exp \left(\frac{i k}{1-\mathrm{M}_{j}} z-2 i k \zeta\right) \\
& +D_{1}\left(r, z / \beta_{j}\right)+O\left(\omega^{2} \ln \omega^{=1}\right) \\
& \phi_{z}(r, z \geq 0)=2 \mathrm{M}_{j}\left[\Gamma \exp \left\{-i \omega\left(\frac{1-\mathrm{M}_{j}}{1+\mathrm{M}_{j}}\right)^{1 / 2} C_{J}\right\}\right. \\
& \left.+i \omega(1-\Gamma)\left(1+M_{j}\right) \sqrt{\overline{d L}}\right] \exp (-i \omega z) \\
& -E_{0}\left(r, z / \beta_{j}\right)+O\left(\omega^{2} \mathrm{M}_{j} \ln \omega^{-1}\right) \\
& \phi_{z}(r, z \leq 0)=\mathrm{M}_{j} \exp \left(-\frac{i k}{1+\mathrm{M}_{j}} z\right) \\
& +\frac{\mathrm{M}_{j}}{1-\mathrm{M}_{j}}\left(1+\mathrm{M}_{j}-2 \mathrm{M}_{j} \Gamma\right) \exp \left(\frac{i k}{1-\mathrm{M}_{j}} z-2 i k \zeta\right) \\
& -E_{1}\left(r, z / \beta_{j}\right)+O\left(\omega^{2} \mathrm{M}_{j} \ln \omega^{=1}\right)
\end{aligned}
$$

with

$$
\begin{gathered}
\zeta=\frac{\left(1+\mathrm{M}_{j}\right)(1-\Gamma)}{1+\mathrm{M}_{j}-2 \mathrm{M}_{j} \Gamma} \sqrt{d L}+\frac{1}{\beta_{j}} C_{J} \\
D_{v}\left(r, z^{\prime}\right)=\frac{2 \mathrm{M}_{j}(1-\Gamma)}{1-\mathrm{M}_{j}} \exp \left(i \Theta\left(z^{\prime}\right)\right) \Sigma_{v}^{\prime}\left(r, z^{\prime}\right) \\
+\frac{2 i k\left(1-\mathrm{M}_{j} \Gamma\right)}{\beta_{j}\left(1-\mathrm{M}_{j}\right)} \Sigma_{v}\left(r, z^{\prime}\right) \\
E_{v}\left(r, z^{\prime}\right)= \\
\frac{2 \mathrm{M}_{j}(1-\Gamma)}{1-\mathrm{M}_{j}} \exp \left(i \theta\left(z^{\prime}\right)\right) \Sigma_{v}^{\prime}\left(r, z^{\prime}\right) \\
+\frac{2 i k}{\beta_{j}}\left[\Gamma+(1-\Gamma) \frac{\mathrm{M}_{j}^{2}}{1-\mathrm{M}_{j}}\right] \Sigma_{v}\left(r, z^{\prime}\right) \\
\theta\left(z^{\prime}\right)=\frac{k \mathrm{M}_{j}}{\beta_{j}} z^{\prime}-\omega\left(1+\mathrm{M}_{j}\right) \sqrt{d L}-\frac{k}{\beta_{j}} C_{J}
\end{gathered}
$$

$$
\Sigma_{v}\left(r, z^{\prime}\right)=\sum_{m=1}^{\infty} \frac{J_{0}\left(r j_{v, m}\right) \exp \left(-j_{v, m}\left|z^{\prime}\right|\right)}{j_{v, m}\left(1+\frac{j_{v, m}}{j_{\tilde{0}, m}}\right) \prod_{\substack{n \neq 1 \\ n \neq m}}^{\infty}\left(1-\frac{j_{v, m}}{j_{v, n}}\right)\left(1+\frac{j_{v, m}}{j_{\tilde{0}, n}}\right)}
$$

(with $\bar{v}=1$ if $v=0, \bar{v}=0$ if $v=1$ ),

$$
\Sigma_{u}^{\prime}\left(r, z^{\prime}\right)=\frac{\partial}{\partial z^{\prime}} \Sigma_{u}\left(r, z^{\prime}\right)
$$

and the constant

$$
C_{J}=0.25538 \ldots=\sum_{n \equiv 1}^{\infty} \frac{1}{\overline{j_{0, n}}}-\frac{1}{\overline{j_{1, n}}}
$$

A numerical evaluation of $\Sigma_{u}$ and $\Sigma_{v}^{\prime}$ is, due to the exponential term, possible, provided $\left|z^{\prime}\right|$ is not too close to zero. If $\left|z^{\prime}\right|=0$ and $r=0$, the series of $\Sigma_{y}$ converges very slowly (like $\left.\Sigma(-1)^{n} / n\right)$, and the series of $\Sigma_{v}^{\prime}$ converges not at all.

In the foregoing expressions for pressure and velocity, the term with $\exp \left(i k\left(1 \equiv \mathbf{M}_{j}\right)^{-1} z\right)$ represents the reflected wave, the term with $\exp (=i \omega z)$ the instability waves (both from $u_{0}$ and $\left.u_{1}\right)$, and the terms $D_{v}$ and $E_{v}$ the exponentially decreasing field of the other, cutoff, modes. Note that (on $z \leq O\left(\omega^{-1}\right)$ ) the instability wave is of purely hydrodynamic nature, and is absent in pressure. A first indication of the energy conversion mechanism by vortex shedding is seen in equation (15) from the rapid increase of this instability amplitude when $\Gamma$ varies from zero to a finite value.

From these expressions, we obtain immediately

-impedance at $z \equiv 0: Z=Z^{*} / \rho_{j} c_{j}=\mathrm{M}_{j} p(r, 0) / \phi_{z}(r, 0)$;

$$
Z=-M_{J}\left\{1=\frac{1=M_{j}}{\Sigma_{0}^{\prime}(r, 0)} \cdot \frac{\Gamma}{1-\Gamma}\right\}^{-1}+O\left(k \ln ^{1 / 2} \omega^{-1}\right)
$$

which becomes for $\Gamma=1$ :

$$
Z=\frac{i k}{\beta_{j}} \Sigma_{0}(r, 0)+O\left(\omega^{2} \ln \omega^{=1}\right)
$$

-reflection coefficient for pressure:

$R=$ reflected wave ampl./primary wave ampl.;

$$
R=-\frac{1+\mathrm{M}_{j}-2 \mathrm{M}_{j} \Gamma}{1-\mathrm{M}_{j}} \exp (=2 i k \zeta)+O\left(\omega^{2} \ln \omega^{-1}\right)
$$

-end correction: point $z=l\left(\bmod \pi \beta_{j}^{2} / k\right)$ where primary and reflected waves are $180 \mathrm{deg}$ out of phase;

$$
\begin{gathered}
l=\beta_{j}^{2} \operatorname{Re}(\zeta)+\frac{\beta_{j}^{2}}{2 k} \arctan \left[\frac{2 \mathrm{M}_{j} \operatorname{Im}(\Gamma)}{1+\mathrm{M}_{j}-2 \mathrm{M}_{j} \operatorname{Re}(\Gamma)}\right] \\
+O\left(\omega M_{j}^{-1} \ln \omega^{-1}\right)
\end{gathered}
$$

We see that for $\Gamma \neq 1$ the field inside the flow is coupled with the ambient flow, viz., through the parameter $d L$. In case of the full Kutta condition $(\Gamma=1)$, this coupling disappears. Another conclusion to be noted is the importance of the choice of $\Gamma$. For instance, in view of the fact that $L$ and, even more, $1 / k$ are large, the end correction can be virtually anything for only slightly varying $\Gamma$. When $\Gamma$ is not known and cannot be kept constant, measurements of the end correction will be very difficult for small $\omega$.

Somewhat surprising may be the fact that for $M_{j} \rightarrow 0$ (and $d=C=1, s=0$ ) the end correction does not tend to 0.6133 , the value found by Levine and Schwinger [14] for the case $\mathbf{M}_{j}=$ $0, k \rightarrow 0$. This is due to the nonuniform character of the limit $\mathrm{M}_{j} \rightarrow 0, k \rightarrow 0$. The Levine and Schwinger case corresponds to $\omega \rightarrow \infty$ (no flow), whereas our case has $\omega \rightarrow 0$ (incompressible flow).

To study the energy balance, we consider the power flux $P^{t *}$ through the pipe. If we write 


$$
\begin{aligned}
& P^{\prime *} \equiv 1 / 4 D^{2} \rho_{j} U j P^{\prime}, \text { then we have [10] } \\
& \left.P^{\prime} \equiv 1 /\left.2 \pi \mathbf{M}_{j}\left|\left(1 \neq \mathbf{M}_{j}\right)^{2}\right| p_{\neq}\right|^{2}=\left(1=M_{j}\right)^{2}\left|p_{=}\right|^{z}\right],
\end{aligned}
$$

where $\left|p_{\neq}\right|$and $\left|p_{=}\right|$are the amplitudes of incident and reflected pressure wave. Hence

$$
\begin{gathered}
P^{\prime} \equiv 1 / 2 \pi \mathrm{M}_{j}\left\{\left(1 \neq \mathrm{M}_{j}\right)^{2}\right. \\
\left.=\left|1 \neq \mathrm{M}_{j}=2 \mathrm{M}_{j} \Gamma\right|^{2} \exp \left[4 k\left(1 \neq \mathrm{M}_{j}\right) \sqrt{\overline{d L}} \operatorname{Im}\left\{\frac{1=\Gamma}{1+\mathrm{M}_{j}=2 \mathrm{M}_{j} \Gamma}\right\}\right]\right\} \\
\neq O\left(\omega k \ln \omega^{=1}\right)
\end{gathered}
$$

Note that $P^{t} \equiv 0$ for $\Gamma \equiv 0$, and that for a real $\Gamma$ the power flux is independent of the ambient medium.

Finally we present the far field approximations

$$
\begin{gathered}
|p| \equiv \frac{d k \mathrm{M}_{j}\left(1 \neq \mathrm{M}_{j}\right)\left(1=\mathrm{M}_{0} \cos \xi\right)^{2}}{2 \lambda\left(1=\mathrm{M}_{j} \operatorname{Ccos} \xi+\mathrm{CM}_{j} \mathrm{M}_{0}\right)^{2}} \mid(1=\Gamma) \mathrm{C}\left(\cos \xi=\mathrm{M}_{0}\right) \\
\neq \frac{\Gamma}{1 \neq \mathrm{M}_{j}}=\tilde{u}_{0} \mid\left(1 \neq O\left(\omega^{2} \ln \omega^{-1}\right)\right)
\end{gathered}
$$

Analogous expressions (differing from this only by a factor) are easily found for the potential and velocity. From these we obtain the far field intensity [10], which yields, after in= tegration, the radiated power flux $P^{r *} \equiv 1 / 4 D^{2} \rho_{j} U U_{j} P^{r}$, by

$$
P^{r} \equiv \frac{\pi \mathrm{M}_{j} C}{d} \beta_{0} \lambda^{z} \int_{0}^{\pi} \frac{\sin \xi}{\left(1=\mathrm{M}_{0} \cos \xi\right)^{2}}|p|^{z} d \xi
$$

Although there is, in principle, no difficulty in ealeulating the general case, the formulas become cumbersome, and we present here only the power flux for the Kutta condition case and for the stable case.

$$
\begin{gathered}
P_{k}^{r} \equiv 1 / 2 \pi d k^{2} \mathrm{M}_{j} C \frac{1 \neq 1 / 3(1=s)^{2} \mathrm{M}_{j}^{2} C^{2}}{\left[1=(1=s)^{\frac{2}{2}} \mathrm{M}_{j}^{2} C^{2}\right]^{3}}\left(1 \neq O\left(\omega^{2} \ln \omega^{=1}\right)\right) \\
P_{s}^{r} \equiv 1 / 2 \pi d k^{2} \mathrm{M}_{j} C\left(1 \neq \mathrm{M}_{j}\right)^{2}\left[\frac{(1=s)^{2}}{\overline{1=(1=s)^{2} \mathrm{M}_{j}^{2} C^{2}}}\right. \\
\left.\neq \frac{s}{\mathrm{M}_{j} C} \ln \frac{1 \neq \mathrm{M}_{j} C}{1=\mathrm{M}_{j} C}\right]\left(1 \neq O\left(\omega^{2} \ln \omega^{=1}\right)\right)
\end{gathered}
$$

The expression for $P_{k}^{r}$ agrees with the one obtained previously by Cargill [5].

For a fixed amplitude of the incident wave, the edge condition does affeet the radiated power, although it does not change the order of magnitude. The important difference appears when we compare radiated and transmitted power, and we recognize from the small ratio

$$
P_{k}^{r} / P_{k}^{\prime} \approx k^{2} d C \frac{1+1 / 3(1=s)^{2} \mathrm{M}_{j}^{2} C^{2}}{\left[1-(1=s)^{2} \mathrm{M}_{j}^{2} C^{2}\right]^{3}}
$$

Bechert and Howe's sound attenuation mechanism.

\section{Discussion}

The cold jet far field formula $|p|$, with Kutta condition, of equation (26) is shown by Cargill [5] to be in very good agreement with Munt's [17] numerical evaluation, and hence with Pinker and Bryce's [19] measurements, for $\omega$ even up to 2. Also in the hot jet case our formula (with $\Gamma=1$ ) is in very good agreement with Munt (Fig. 3), even in the cases where it should not, viz., where $M_{j} C \geq 1$ and $\omega$ is not small. Only the density ratio $d$ seems to be an important parameter; $d=1$ gives best agreement, while $d \simeq 2.8$ is too high. As noted by Munt, a comparison with experiments is, for the hot jet, not so successful (Fig. 3). In general, the difference between the downstream and the upstream are is over-predicted, and the measurements tend to behave more like a $|p|$ with $\Gamma$ closer to zero; however, a satisfactory fit was not readily found. This

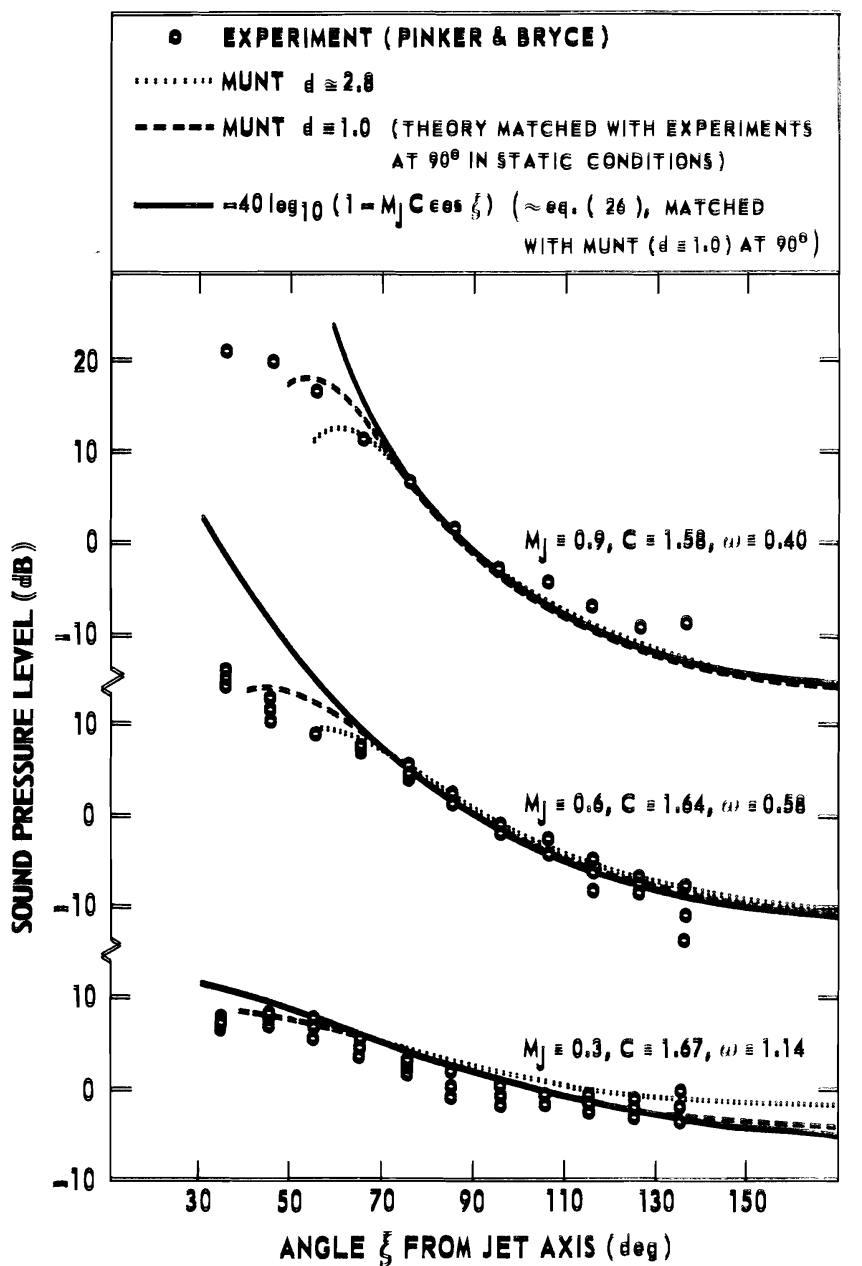

Fle. 3 Comparleon of far fleld $|p|$ (equation (26), $\Gamma=1$ ) with numerical ovaluatlon (Munt) and experlments (PInker and Bryeo) for a hot statle Jot $(0=0)$ at $f^{*}=2.5 \mathrm{kHz}$ and $a$ (total) Jet temperature of $830^{\circ} \mathrm{K}$

behavior may indicate that, by some as yet unknown temperature effect, the Kutta condition is not satisfied, and the analytical solution would improve on using some $\Gamma$ smaller than unity. Also for the cold jet it was found experimentally by Heavens [11] that for high excitation levels the diffracted field increased more than proportionally in amplitude, also indicating a deviation from the Kutta condition.

For small $k$, formula (28) of the sound energy loss by vortex shedding covers very well the cold jet experimental data of Bechert, et al. [2], as shown by Cargill [5]. No data for a hot jet are known.

Also of the field inside the jet flow, few measurements are known. Moore [15] did, in a cold jet, measurements for $\omega \geq$ 0.56 . His measurements of the axial variation of the pressure level on the jet centerline are compared with the predictions of our formulas (13) and (14) for $\Gamma=1$ (Kutta condition) in Fig. 4. Inside the pipe, agreement is reasonable; the position and the "depth" of the minima confirm the assumption of the Kutta condition being adequate. (An experimental value of $\Gamma$ can be derived from the difference between maximum and estimated minimum; if we assume $\Gamma$ real, all those results of Moore with $\omega<1$, viz., $\omega \equiv 0.56,0.70,0.89$, and 0.94 , yield, surprisingly, identical values, namely, $\Gamma=0.96$ ). Outside the pipe, however, agreement is, if not absent, limited to the exit vicinity. The instability wave always dominates beyond $z=$ $1 / 2$.

No published measurements are known to the author on the end correction and on the reflection coefficient for a hot jet. In case of a cold jet (e.g., [24], our value $|R| \equiv 1$ for the Kutta condition case is indeed well approached for $\omega \rightarrow 0$ by the experiments; also Munt's [18] numerical evaluation comes 
to that value. Measurements on the end correction seem to suffer often from two much seatter to permit decisive con= clusions. Possibly this is due to a sensitiveness to the edge condition. From expression (24) we see that the products $(1=$ $\Gamma), \bar{d} L$ and $\operatorname{Im}(\Gamma) / k$ give order one (and more) variations in $l$ for small variations of $\Gamma$. Especially a phase lag of the in= stability wave (giving a nonzero imaginary part of $\Gamma$ ) seems very effective.

Be that as it may, further work is clearly required to establish the domains of applicability of Kutta condition, and other choices of $\Gamma$. Experiments $[2=11]$ as well as theoretical work [4] indicate a genuine dependence of $\Gamma$ on Strouhal number, amplitude, Reynolds number, and other parameters; systematic experiments in particular are now needed to find $I$ (or an analogous parameter if the present model is too crude) as function of $\omega, \mathbf{M}$, etc., and to relate far field and the field inside the pipe for varying $\Gamma$.

Finally, it may be noted that in view of the nonuniform behavior of the complete solution in the limit $\left(k, \mathbf{M}_{j}\right) \Rightarrow(0,0)$, it may be worthwhile to present, in case of a small $k$ and small $\mathrm{M}_{j}$, experimental data as a function of Strouhal number instead of Helmholtz number, the more usual parameter.

\section{Conclusions}

A small Strouhal number $\omega$ asymptotic analysis has been made of a model, describing harmonic plane sound waves radiating out of an exhaust pipe, with density and temperature differences inside and outside, and a slowly moving ambient medium. Munt's [17] acoustically exact solution was adapted to satisfy an arbitrary pipe edge condition, and this general solution was systematically approximated, using complex contour deformation, with a relative accuracy of $O\left(\omega^{2} \ln \omega^{-1}\right)$. Velocity and pressure field inside the jet flow and in the far field, impedance, reflection coefficient and end correction at the pipe mouth, and transmitted and radiated sound power were derived.

The role of the edge condition is shown to be always significant and for some quantities very important. The effect of deviations from the Kutta condition is discussed and argued to be a possible source of present experimental discrepancies and scatter, particularly in the end correction ( phase of the reflection coefficient). A curious observation is the independence of the field inside the pipe flow from the ambient medium properties just (only) in case of the Kutta condition. Time seems ripe for experimental investigations on the relation between the edge condition, as depending on flow parameters, and the sound field.

Finally, the exact solution is shown to behave nonuniformly in the limit of Mach number and Helmholtz number both to zero.

\section{References}

1 Bechert, D. W., "Sound Absorption Caused by Vorticity Shedding, Demonstrated with a Jet Flow," AIAA 5th Aeroacoustics Conference, Mar. 1979, Paper No. 79-0575.

2 Bechert, D. W., Michel, U., and Pfizenmaier, E., "Experiments on the Transmission of Sound through Jets," AIAA 4th Aeroacoustics Conference, Oct. 1977, Paper No. 77-1278.

3 Bechert, D. W., and Pfizenmaier, E., "Optical Compensation Measurements on the Unsteady Exit Condition at a Nozzle Discharge Edge," Journal of Fluid Mechanics, Vol. 71, Part 1, 1975, pp. 123-144.

4 Brown, S. N., and Daniels, P. G., "On the Viscous Flow about the Trailing Edge of a Rapidly Oscillating Plate," Journal of Fluid Mechanics, Vol. 67, Part 4, 1975, pp. 743-761.

5 Cargill, A, M., "Low Frequency Sound Radiation Due to the Interaction of Unsteady Flows with a Jet Pipe," Proceedings of the Symposium on Mechanics of Sound Generation in Flows, IUTAM/ICA/AIAA, Göttingen, Aug. 1979; Berlin, Springer-Verlag.

6 Crighton, D. G., "The Excess Noise Field of Subsonic Jets," Journal of Fluid Mechanics, Vol. 56, Part 4, 1972, pp. 683-694.

7 Crighton, D. G., "Why Do the Acoustics and the Dynamics of a Hypothetical Mean Flow Bear on the Issue of Sound Generation by Turbulence?," Proceedings of the Symposium on Mechanics of Sound Generation in Flows, IUTAM/ICA/AIAA, Göttingen, Aug. 1979; Berlin, SpringerVerlag.
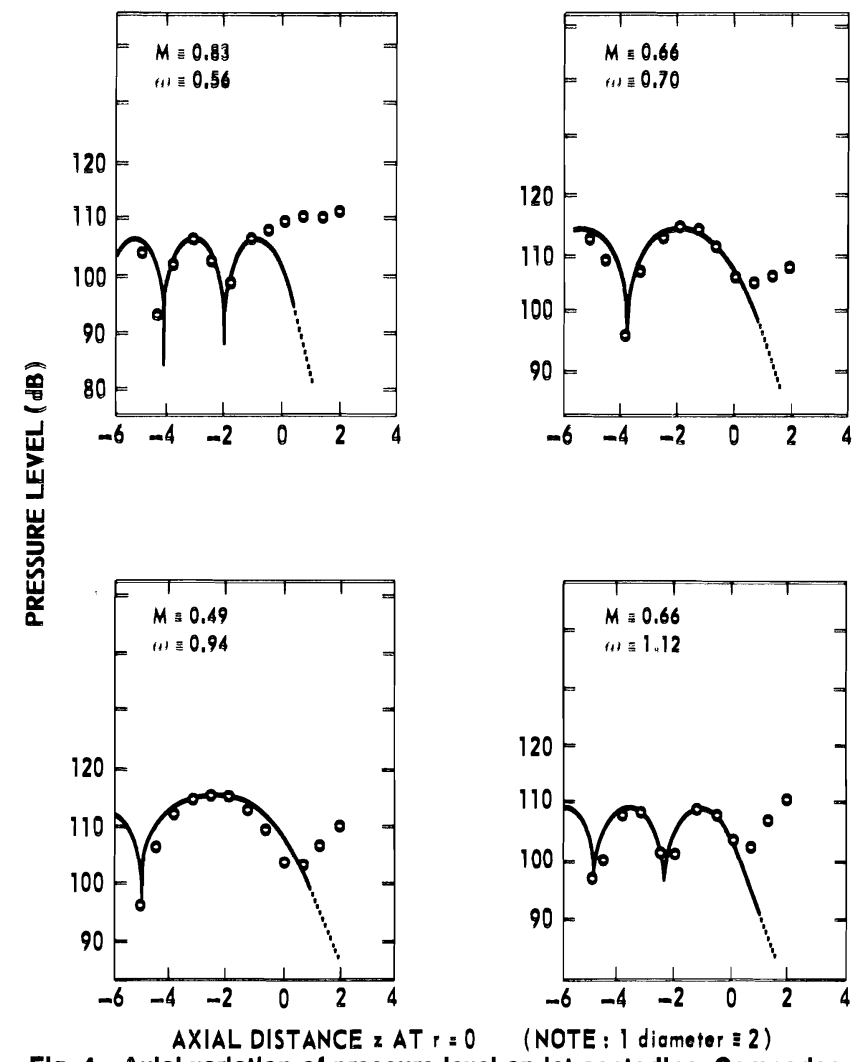

(NOTE : 1 diameter $\equiv 2$ )

Flg. 4 Axlal varlation of pressure level on Jet centerline. Comparlson between experiments (0, Moore's [15] Flg. 20) and equatlons (13) and (14). Cold Jet without coflow $(\Gamma=C=d=1, s=0)$; matched at maximum level In duct

8 Crighton, D. G., and Leppington, F. G., "Radiation Properties of the Semi-Infinite Vortex Sheet: the Initial Value Problem," Journal of Fluid Mechanics, Vol. 64, Part 2, 1974, pp. 393-414.

9 Ffowes Williams, J. E., "Aeroacoutics," Annual Review of Fluid Mechanics, Vol. 9, 1977, pp. 447-468.

10 Goldstein, M. E., Aeroacoustics, MeGraw-Hill, New York, 1976.

11 Heavens, S. N., "Acoustic Excitation of a Subsonic Jet," Unpublished report Whittle Laboratory, University of Cambridge, England, 1979.

12 Howe, M. S., "Attenuation of Sound in a Low Mach Number Nozzle Flow," Journal of Fluid Mechanics, Vol. 91, Part 2, 1979, pp. 209-229.

13 Jones, D. S., and Morgan, J. D., "The Instability of a Vortex Sheet on a Subsonic Stream under Acoustic Radiation," Proceedings Cambridge Philosophical Society, Vol. 72, 1972, pp. 465-488.

14 Levine, H., and Schwinger, J., "On the Radiation of Sound from an Unflanged Circular Pipe," Physical Review, Vol. 73, No. 4, 1948, pp. 383-406.

15 Moore, C. J., "The Role of Shear-layer Instability Waves in Jet Exhaust Noise," Journal of Fluid Mechanics, Vol. 80, Part 2, 1977, pp. 321-367.

16 Morgan, J. D., "The Interaction of Sound with a Semi-Infinite Vortex Sheet," Quarterly Journal of Mechanics and Applied Mathematics, Vol. 27, Part 4, 1974, pp. 465-487.

17 Munt, R. M., "The Interaction of Sound with a Subsonic Jet Issuing from a Semi-Infinite Cylindrical Pipe," Journal of Fluid Mechanics, Vol. 83, Part 4, 1977, pp. 609-640.

18 Munt, R. M., "Acoustic Transmission Properties of a Jet Pipe with Subsonic Jet Flow," Journal of Sound and Vibration, 1980.

19 Pinker, R. A., and Bryce, W. D., "The Radiation of Plane-Wave Duct Noise from a Jet Exhaust, Statically and in Flight," AIAA 3rd Aeroacoustics Conference, July 1976, Paper No. 76-581.

20 Rienstra, S. W., "Edge Influence on the Response of Shear Layers to Acoustic Forcing,"'Ph.D. thesis, June 1979, Technical University Eindhoven, The Netherlands.

21 Rienstra, S. W., "Sound Diffraction at a Trailing Edge," submitted for publication to Journal of Fluid Mechanics, 1980.

22 Rienstra, S. W., "A Small Strouhal Number Analysis for Acoustic Wave-Jet Flow-Pipe Interaction," submitted for publication to Journal of Fluid Mechanics, 1980

23 Savkar, S. D., "Radiation of Cylindrical Duct Acoustic Modes with Flow Mismatch," Journal of Sound and Vibration, Vol. 42, Part 3, 1975, pp. 363-386.

24 Schlinker, R. H., "Transmission of Acoustic Plane-Waves at a Jet Exhaust," AIAA 15th Aerospace Sciences Meeting, Jan. 1977, Paper No. 77-22.

25 Watson, G. N., A Treatise on the Theory of Bessel Functions, 2nd Ed., Cambridge University Press, 1966. 


\section{E R R A T A}

Corrections to "On the Acoustical Implications of Vortex Shedding from an Exhaust Pipe," by Dr. S. W. Rienstra, ASME JoURNAL OF ENGINEERING FOR INDUSTRY, November 1981, Vol. 103, No. 4, pp. 378-384.

The following typographical errors should be noted in the above paper:

- Equation (10) should be divided as follows:

$\ldots \mu_{-}\left(\frac{1}{1+M_{j}}\right) \ldots$

- The equation just below equation (12) should read:

$\mu(u)=\frac{\left(1-u \mathrm{M}_{j}\right)^{2} \ldots}{\ldots}$

- Equation (15) should read:

$\ldots \exp \left\{i \omega\left(\frac{1-\mathrm{M}_{j}}{1+\mathrm{M}_{j}}\right)^{1 / 2} C_{J}\right\} \ldots$

- Equation (28) should read:

$P_{k} / P_{k}=\frac{k^{2} d C}{4 \mathrm{M}_{j}} \frac{\ldots}{\ldots}$ 